Review Article

\title{
Exploring Heart Rate Variability as a Biomedical Diagnostic Tool for the Disympathetic Dimension of Eight-Constitution Medicine
}

\author{
Hyonna Kang $\mathbb{D}^{1,2}$ Sean Walsh $\mathbb{D}^{1},{ }^{1}$ Brian Oliver $\mathbb{D}^{1},{ }^{1,3}$ Terry Royce $\mathbb{D}^{\mathbb{D}},{ }^{4}$ and Byung Je Cho ${ }^{5,6}$ \\ ${ }^{1}$ School of Life Sciences, University of Technology Sydney, Sydney, NSW, Australia \\ ${ }^{2}$ Sage Acupuncture, Sydney, NSW, Australia \\ ${ }^{3}$ Woolcock Institute of Medical Research, The University of Sydney, Sydney, NSW, Australia \\ ${ }^{4}$ Graduate Research School, University of Technology Sydney, Sydney, NSW, Australia \\ ${ }^{5}$ Chedam Hospital of Korean Medicine, Busan, Republic of Korea \\ ${ }^{6}$ Korean Medicine, Dong-Eui University, Busan, Republic of Korea
}

Correspondence should be addressed to Hyonna Kang; hyonna@sageacupuncture.com.au

Received 7 October 2020; Revised 26 November 2020; Accepted 31 May 2021; Published 16 June 2021

Academic Editor: Christopher Worsnop

Copyright (C) 2021 Hyonna Kang et al. This is an open access article distributed under the Creative Commons Attribution License, which permits unrestricted use, distribution, and reproduction in any medium, provided the original work is properly cited.

Background. Eight-Constitution Medicine (ECM), an extension of Traditional Korean Medicine, divides the population into eight groups based on their physiological characteristics. ECM divides these eight groups into two larger groups based on autonomic reactivity: the Sympathicotonic group and the Vagotonic group (herein referred to as the Disympathetic Dimension). Heart Rate Variability (HRV) is a widely used biomedical tool to assess cardiac autonomic function. This raises the question of the utility of using HRV to correctly diagnose ECM constitutions. Methods. A systematic literature review was conducted to evaluate the correlation between HRV and constitutions in Korean Constitutional Medicine, including Eight-Constitution Medicine (ECM) and Sasang Constitution Medicine (SCM). The articles were obtained from both English (Scopus, PubMed, EMBASE, ProQuest, and Medline) and Korean databases (NDSL and RISS), in addition to Google Scholar, without date restriction. 20 studies met the inclusion criteria, and data were extracted against three aspects: (1) correlation between HRV and constitution, (2) HRV reporting and interpretation, and (3) extraneous factors that were controlled in the studies. Results. 386 articles were initially identified, which was reduced to $n=20$ studies which met the inclusion criteria. Of these, 19 were SCM studies and 1 was an ECM study. Sample sizes varied from 10 to 8498 men and women, with an age range of 10-80 years. SCM studies explored HRV differences by constitution, measuring HRV at resting, with controlled breathing, before and after acupuncture stimulation, and by other interventions. SCM studies reported either no significant differences (HRV at resting or with controlled breathing studies) or conflicting data (HRV with acupuncture stimulation studies). The single ECM study measured HRV at resting and after acupuncture stimulation but reported no significant differences between the two groups of Sympathicotonia and Vagotonia. Conclusions. Due to inconsistencies in study design, study population, and measures of HRV, there was no consistency in the data to support the use of HRV as a biomedical determinant of ECM constitutions.

\section{Introduction}

Eight-Constitution Medicine (ECM) originates from Korean Constitutional Medicine, a further development of Sasang Constitution Medicine (SCM) [1-3]. A constitution refers to the nature of an individual's health response based on their psychosocial and physiological traits. While SCM classifies people into one of four constitutions (Tae-Yang, Tae-Eum, So-Yang, and So-Eum) [4], ECM differentiates people as one of the eight constitutions: Pulmotonia (PUL), Colonotonia (COL), Renotonia (REN), Vesicotonia (VES), Pancreotonia (PAN), Gastrotonia (GAS), Hepatonia (HEP), and Cholecystonia (CHO) (Figure 1) [5]. Consequently, ECM employs a personalized approach to treatment, even between 

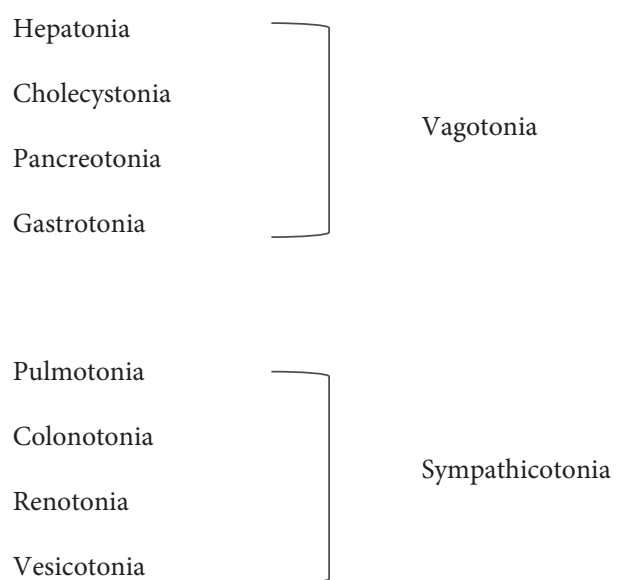

FIGURE 1: Eight constitutions and Disympathetic Dimension [5].

people with the same 'disease', prescribing individualized neuromodulatory protocols (via acupuncture) and lifestyle regimen (including dietary guidance), aligning with the emerging "personalized and preventive" medicine movement [6-8].

However, despite over 50 years of ECM research in Korea [2, 5, 9-11], differentiating a patient's constitution still primarily relies upon the practitioner's assessment of the radial arterial pulse. This requires highly developed palpatory skills to detect distinct differences in pulse position and contours that differentiate one constitution from another [12-15]. While there is greater interrater agreement reported between experienced practitioners, agreement levels, unfortunately, reduce among inexperienced practitioners $[13,14,16,17]$.

To better support reliability and remove subjectivity, a proposal is to differentiate constitutions based instead on autonomic balance. Eppinger and Hess introduced the constitutional concepts of Vagotonia and Sympathicotonia [18], which have a relationship with Heart Rate Variability (HRV). They defined Sympathicotonia as having increased tone in the sympathetic nervous system and with an abnormal increased response to adrenalin, and Vagotonia as having increased tone in the parasympathetic nervous system and with a relatively increased sensitiveness to pilocarpine $[5,18,19]$. This aligns with ECM, which proposes these hereditary factors as constitutional differences and classifies four (of the eight) constitutions into Sympathicotonic type and Vagotonic type (Figure 1) [5].

A set of biomedical diagnostics that differentiates the Sympathicotonic and Vagotonic types of ECM (referred to herein as the 'Disympathetic Dimension') would provide objective support for assessing the eight-constitution framework. Furthermore, HRV is a widely used biomedical tool to objectively assess cardiac autonomic function [20]. While it is generally agreed that high-frequency HRV can be used to assess cardiac vagal modulation (parasympathetic) [20-25], the same cannot be said for low-frequency HRV assessing cardiac sympathetic modulation [26-31]. HRV, however, is very sensitive to a range of extraneous factors [32].
Consequently, to explore HRV as a biomedical diagnostic for ECM, HRV studies in the Korean Constitutional Medicine (Eight-Constitution Medicine, Sasang Constitution Medicine) were critically reviewed against three considerations: (1) the correlation between HRV and constitutions, (2) HRV reporting and interpretation, and (3) controlled extraneous factors.

\section{Methods}

2.1. Databases. A systematic review was conducted on fulltext articles obtained from both English (Scopus, PubMed, EMBASE, ProQuest, and Medline) and Korean (NDSL, RISS) electronic databases, in addition to Google Scholar, without date restriction.

2.2. Search Terms. Search terms for English databases include ("heart rate variability" OR HRV) AND "eight constitution", ("heart rate variability" OR HRV) AND "8 constitution*", ("heart rate variability" OR HRV) AND "Sasang", while Korean databases search terms include: "heart rate variability" AND 8체질, HRV AND 8체질, HRV AND 팔체질, 심박* AND 팔체질, 심박* AND 8체질, "Heart rate variability" AND 사상체질, "HRV" AND 사상 체질, 심박* AND 사상체질.

\section{Results}

3.1. Review Process. From the 386 total records obtained from database search $(n=384)$ and manual searches $(n=2)$, full-text articles of $n=36$ were obtained after excluding duplicated papers $(n=60)$ and nonrelevant papers or unavailable articles $(n=290)$. The articles $(n=36)$ were further reviewed against the inclusion criteria (i.e., short-term recordings of HRV) for Korean Constitutional Medicine (Eight Constitution or Sasang Constitution). A further 16 articles were excluded, leaving $n=20$ papers for critical review. Of these, one was an ECM article, and the others were SCM studies $(n=19)$. The review process is presented in Figure 2.

\subsection{Study Characteristics (Table 1)}

3.2.1. Demographic Characteristics. Sample sizes varied from 10 to 8498 men and women, with an age range of 10 to 80 years. 13 out of 20 studies were in healthy subjects, and the rest were either patient populations or medical information not being available.

3.2.2. Study Intervention. To explore constitutional differences, the studies measured HRV at resting level [40, 43, 45, 53] with paced breathing $[34,36,54]$, after acupuncture stimulation $[38,39,41,44,47,51,52]$, or other interventions such as meditation [37], forest healing program [42], autogenic training [48], emotional stimulus [50], and constitutional herbal formula [46]. 

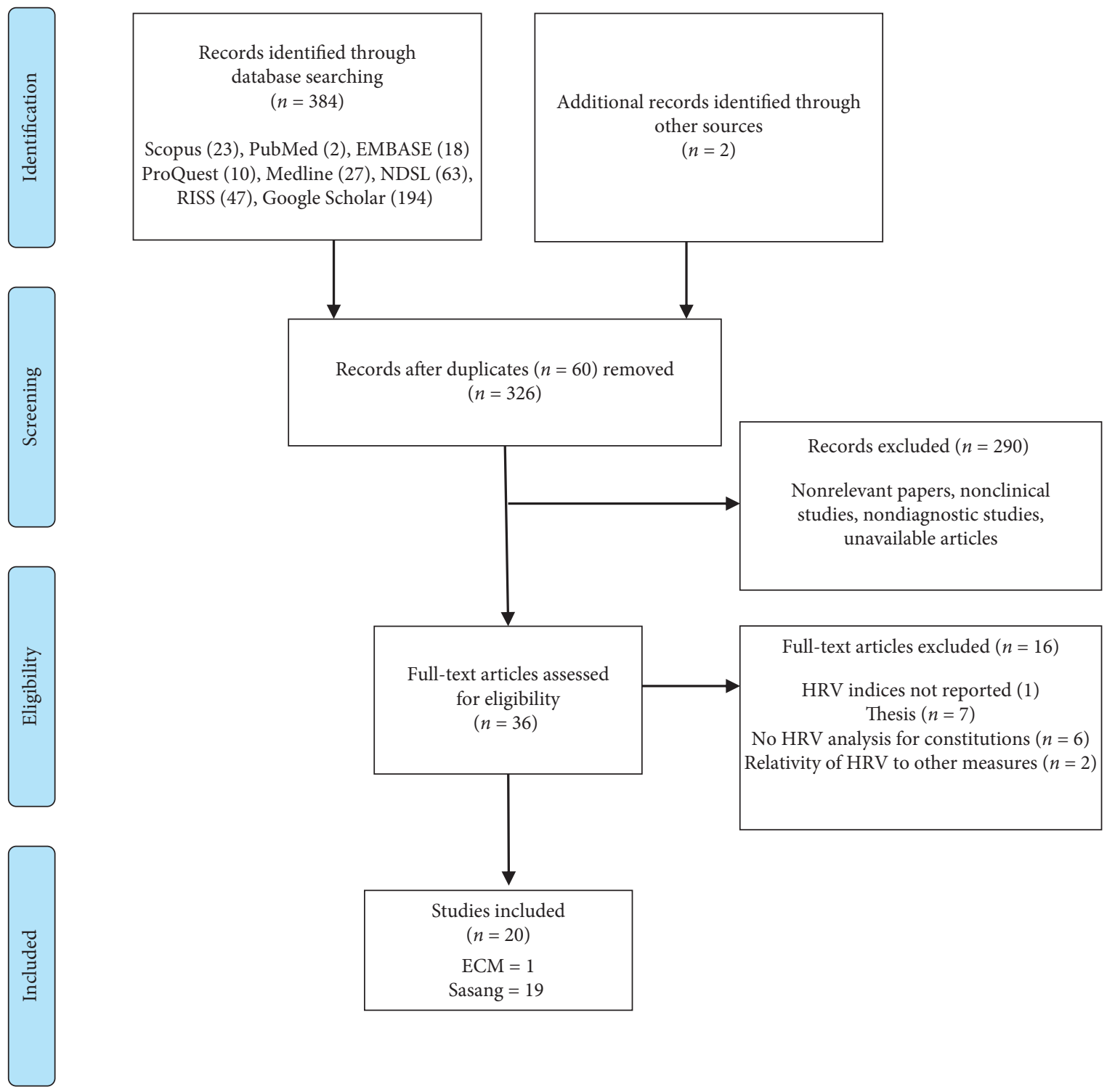

Figure 2: Systematic review process.

3.2.3. HRV Analysis and Devices. HRV analysis studies varied: time and frequency domain $(n=15)$, frequency domain only $(n=4)$, and time domain only $(n=1)$. All studies used commercial HRV medical devices of ECG $(n=16)$, PPG $(n=2)$, or IBI $(n=2)$.

\subsection{Correlation between HRV and Constitution}

3.3.1. ECM and HRV at Resting and after Acupuncture Stimulation $(p<0.05)$ (Table 2). A single ECM study [33] measured HRV baseline at resting and after constitutional acupuncture (i.e., a predefined acupuncture formula for a specific constitution) stimulation but reported no significant differences between the two groups of Sympathicotonia and Vagotonia. The study had a small sample size (42 patients), wide age range (14-73 yr), uncontrolled gender factors, and a short observation period after acupuncture.
3.3.2. SCM and HRV at Resting $(p<0.05)$ (Table 3). None of the SCM studies reported significant differences in $\mathrm{HF}$ at resting between constitutions. Two relatively wellcontrolled SCM studies indicated Tae-Eum constitution (with characteristics of increased parasympathetic reactivity) showed a lower LF/HF ratio than the So-Yang constitution (with both parasympathetic and sympathetic reactivity) at resting condition $(p<0.05)[43,47,55]$.

3.3.3. SCM and HRV with Controlled Breathing $(p<0.05)$ (Table 4). Three SCM studies explored the effects of different breathing approaches on constitutions by measuring HRV: breath-counting meditation [36], paced breathing (3, 6 , or 12 times per min) [34], and the ratio of inhalation and exhalation ( $4: 6$ and $6: 4$, respectively) with posture changes [35], but HRV measures from both baseline and controlled breathing showed no difference between constitutions. 
TABLE 1: Summary of studies investigating the correlation between Heart Rate Variability and Korean Constitution Medicine (ECM or SCM).

\begin{tabular}{|c|c|c|c|c|c|c|}
\hline Reference & No. & Medicine & $\begin{array}{l}\text { Population (age } \\
\text { range) }\end{array}$ & Autonomic stimulus & $\begin{array}{l}\text { Duration and } \\
\text { HRV measures }\end{array}$ & Other measures \\
\hline [33] & 1 & ECM & $\begin{array}{l}42 \text { patients } \\
\text { (14-73) }\end{array}$ & Eight-constitution acupuncture & $\begin{array}{l}5 \text { min, frequency } \\
\text { domain }\end{array}$ & BMI \\
\hline$[34]$ & 2 & SCM & $\begin{array}{l}32 \text { healthy } \\
\text { students }(20-30)\end{array}$ & $\begin{array}{l}\text { Paced breathing in specific } \\
\text { respiration rate }\end{array}$ & $\begin{array}{l}5 \text { min, time and } \\
\text { frequency } \\
\text { domain }\end{array}$ & Respiration rate \\
\hline$[35]$ & 3 & SCM & $\begin{array}{l}60 \text { healthy } \\
\text { students }(20-30)\end{array}$ & $\begin{array}{l}\text { Ratio of inhalation and } \\
\text { exhalation, posture (sitting, } \\
\text { standing) }\end{array}$ & $\begin{array}{l}\text { Time and } \\
\text { frequency } \\
\text { domain }\end{array}$ & $\begin{array}{l}\text { Self-evaluation for physical condition (scale } \\
\qquad 10 \mathrm{~cm} \text { ) }\end{array}$ \\
\hline$[36]$ & 4 & SCM & $\begin{array}{c}78 \text { healthy } \\
\text { students }(20-30)\end{array}$ & Breath-counting meditation & $\begin{array}{l}5 \text { min, time and } \\
\text { frequency } \\
\text { domain }\end{array}$ & $\begin{array}{l}\text { Skin conductance, temperature, abdominal } \\
\text { amplitude, thoracic amplitude }\end{array}$ \\
\hline [37] & 5 & SCM & 78 students & Meditation program ( $\alpha$ version) & $\begin{array}{l}\text { Time and } \\
\text { frequency } \\
\text { domain }\end{array}$ & $\begin{array}{l}\text { BDI (depression), STAXI (anger), STAI } \\
\text { (anxiety) questionnaires }\end{array}$ \\
\hline [38] & 6 & SCM & $\begin{array}{l}16 \text { healthy TE } \\
\text { constitution } \\
(20-60)\end{array}$ & Taegeuk acupuncture & $\begin{array}{l}5 \text { min, frequency } \\
\text { domain }\end{array}$ & None \\
\hline [39] & 7 & SCM & $\begin{array}{l}6 \text { healthy SE } \\
\text { constitution men } \\
(20-30)\end{array}$ & Taegeuk acupuncture & $\begin{array}{l}5 \text { min, frequency } \\
\text { domain }\end{array}$ & None \\
\hline$[40]$ & 8 & SCM & $\begin{array}{l}63 \text { fatigue and } \\
\text { nonfatigue } \\
\text { subjects }(40-60)\end{array}$ & None & $\begin{array}{l}5 \text { min, time and } \\
\text { frequency } \\
\text { domain }\end{array}$ & $\begin{array}{l}\text { BMI, biochemistry analysis, pulse wave } \\
\text { analysis, nail fold capillary microscopy, } \\
\text { questionnaires (FSS, GSRS, SF-MPQ, PSQI, } \\
\text { SF-12) }\end{array}$ \\
\hline [41] & 9 & SCM & $\begin{array}{l}8 \text { healthy SY } \\
\text { constitution } \\
\text { women }(20-30)\end{array}$ & Taegeuk acupuncture & $\begin{array}{l}5 \text { min, frequency } \\
\text { domain }\end{array}$ & $x_{0}$ \\
\hline$[42]$ & 10 & SCM & $\begin{array}{c}47 \text { healthy } \\
\text { subjects }(29-66)\end{array}$ & $\begin{array}{l}\text { Forrest healing program } \\
\text { (aroma, foods, tea by } \\
\text { constitution + trekking) }\end{array}$ & $\begin{array}{l}5 \text { min, time and } \\
\text { frequency } \\
\text { domain }\end{array}$ & $\begin{array}{l}\text { BMI, body temperature, vital sign (BP, } \\
\text { SpO2), electroencephalography, } \\
\text { biochemistry analysis, blood cell count, } \\
\text { stress hormone test }\end{array}$ \\
\hline$[43]$ & 11 & SCM & $\begin{array}{l}665 \text { subjects } \\
\quad(39-72)\end{array}$ & None & $\begin{array}{l}5 \text { min, time and } \\
\text { frequency } \\
\text { domain }\end{array}$ & $\begin{array}{l}\text { BMI, BP, fasting blood sugar, cholesterol, } \\
\text { abdominal obesity }\end{array}$ \\
\hline$[44]$ & 12 & SCM & $\begin{array}{c}20 \text { healthy } \\
\text { subjects }(18-30)\end{array}$ & Bee venom acupuncture & $\begin{array}{l}5 \text { min, time and } \\
\text { frequency } \\
\text { domain }\end{array}$ & Pulse wave analysis, cerebral blood flow \\
\hline$[45]$ & 13 & SCM & $\begin{array}{l}103 \text { idiopathic } \\
\text { facial palsy } \\
\text { patients }(10-79)\end{array}$ & None & $\begin{array}{l}5 \text { min, time and } \\
\text { frequency } \\
\text { domain }\end{array}$ & Facial electromyography \\
\hline$[46]$ & 14 & SCM & $\begin{array}{l}10 \mathrm{TE} \\
\text { constitution } \\
\text { patients }\end{array}$ & $\begin{array}{c}\text { Herbal formula for TE } \\
\text { constitution (Jowisengcheong- } \\
\text { tang) }\end{array}$ & $\begin{array}{l}5 \text { min, time and } \\
\text { frequency } \\
\text { domain }\end{array}$ & None \\
\hline [47] & 15 & SCM & $\begin{array}{l}30 \text { healthy men } \\
(20-26)\end{array}$ & Acupuncture at LI4 & $\begin{array}{l}5 \text { min, time and } \\
\text { frequency } \\
\text { domain }\end{array}$ & None \\
\hline$[48]$ & 16 & SCM & $\begin{array}{l}39 \text { patients } \\
(20-59)\end{array}$ & Autogenic training & $\begin{array}{l}5 \text { min, time and } \\
\text { frequency } \\
\text { domain }\end{array}$ & $\begin{array}{l}\text { MBTI questionnaire (extraversion, } \\
\text { introversion) }\end{array}$ \\
\hline [49] & 17 & SCM & 8498 workers & None & $\begin{array}{l}5 \text { min, time and } \\
\text { frequency } \\
\text { domain }\end{array}$ & None \\
\hline$[50]$ & 18 & SCM & $\begin{array}{c}44 \text { healthy } \\
\text { subjects }(20-30)\end{array}$ & $\begin{array}{l}\text { Emotional stimulus (horror } \\
\text { film) }\end{array}$ & $\begin{array}{l}120 \mathrm{sec}, 197 \mathrm{sec} \\
120 \mathrm{sec}, \text { time and } \\
\text { frequency } \\
\text { domain }\end{array}$ & None \\
\hline [51] & 19 & SCM & $\begin{array}{l}86 \text { subjects } \\
(22-25)\end{array}$ & Electroacupuncture & $\begin{array}{c}30 \mathrm{sec}, \text { time } \\
\text { domain }(\mathrm{SDNN})\end{array}$ & None \\
\hline$[52]$ & 20 & SCM & $\begin{array}{l}19 \text { healthy } \\
\text { subjects }\end{array}$ & Acupuncture at LI4 and LR3 & $\begin{array}{l}5 \text { min, time and } \\
\text { frequency } \\
\text { domain }\end{array}$ & BP, BMI \\
\hline
\end{tabular}

ECM, Eight-Constitution Medicine; SCM, Sasang Constitution Medicine; CM, Constitution Medicine; HRV, Heart Rate Variability; MHR, mean heart rate; BMI, body mass index; TE, Taeumin constitution; SY, Soyangin constitution; FSS, Fatigue Severity Scale; GSRS, Gastrointestinal Symptom Rating Scale; SFMPQ, Short-Form McGill Pain Questionnaire; PSQI, Pittsburgh Sleep Quality Index; SF-12, Short-Form Health Survey; BP, blood pressure; SpO 2 , peripheral capillary oxygen saturation; APG, Accelerated Plethysmogram; MBTI, Myers-Briggs Type Indicator. 


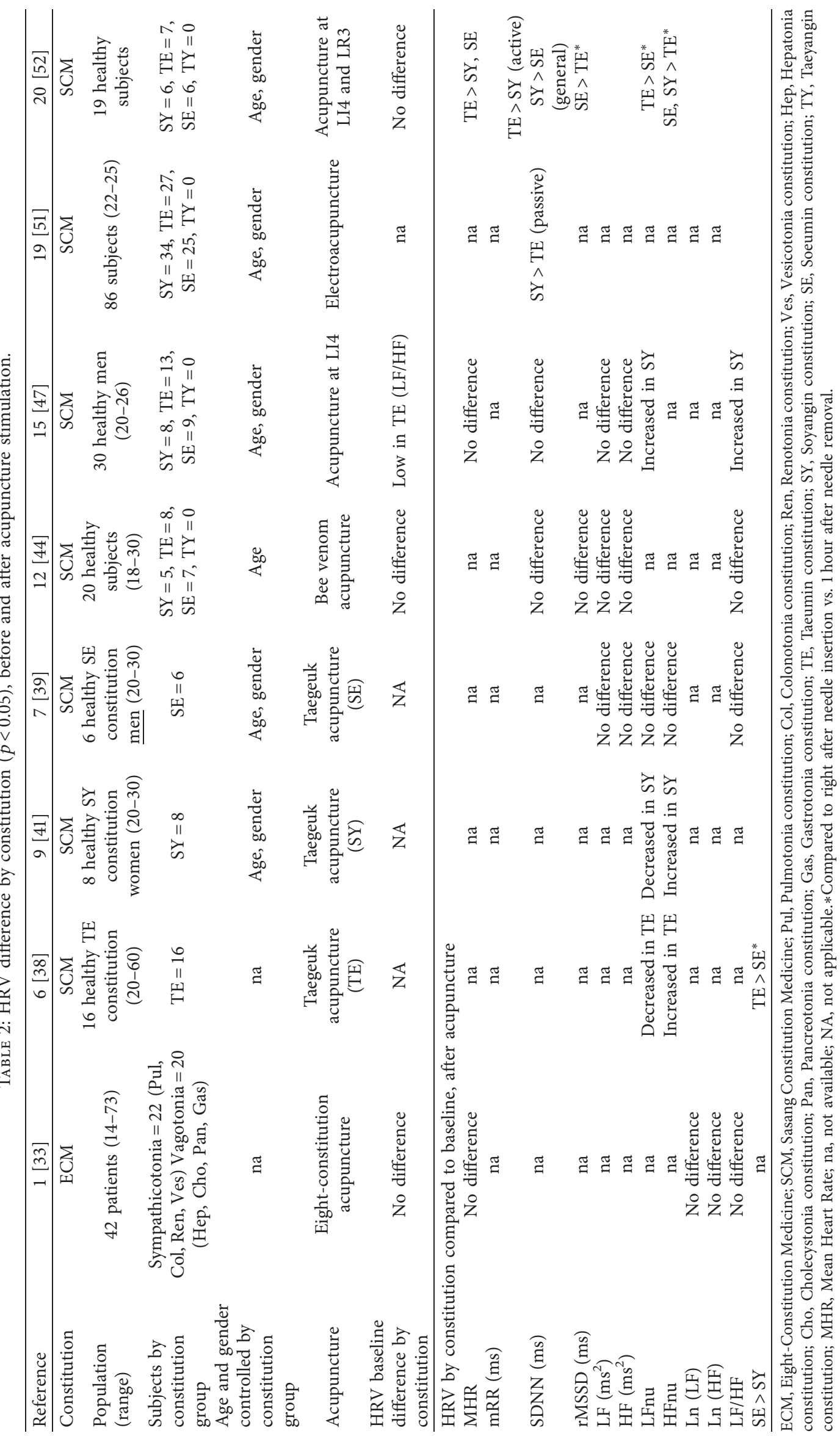


TABLE 3: HRV difference by constitution $(p<0.05)$, at resting.

\begin{tabular}{|c|c|c|c|c|c|}
\hline Reference & $8[40]$ & $11[43]^{1}$ & $13[45]$ & $17[53]^{2}$ & 15 [47] \\
\hline Constitution & SCM & SCM & SCM & SCM & SCM \\
\hline Population (age) & $\begin{array}{c}63 \text { fatigue and } \\
\text { nonfatigue subjects } \\
(40-60)\end{array}$ & 665 subjects $(39-72)$ & $\begin{array}{l}103 \text { idiopathic facial } \\
\text { palsy patients }(10-79)\end{array}$ & 8498 workers & $\begin{array}{l}30 \text { healthy men } \\
(20-26)\end{array}$ \\
\hline $\begin{array}{l}\text { Subjects by } \\
\text { constitution group }\end{array}$ & $\begin{array}{c}\text { Fatigue: } \\
\mathrm{SY}=10, \mathrm{TE}=8, \\
\mathrm{SE}=14, \mathrm{TY}=0 \\
\text { Nonfatigue: } \\
\mathrm{SY}=15, \mathrm{TE}=7, \\
\mathrm{SE}=9, \mathrm{TY}=0\end{array}$ & $\begin{array}{c}\text { Total: } \\
\mathrm{SY}=100, \mathrm{TE}=363, \\
\mathrm{SE}=202, \mathrm{TY}=0 \\
\text { Female }<60 \mathrm{yrs} \\
\mathrm{SY}=36, \mathrm{TE}=140 \\
\mathrm{SE}=68, \mathrm{TY}=0\end{array}$ & $\begin{array}{c}\mathrm{SY}=25(\mathrm{M} 7, \mathrm{~F} 18) \\
\mathrm{TE}=54(\mathrm{M} 27, \mathrm{~F} 27) \\
\mathrm{SE}=24(\mathrm{M} 8, \mathrm{~F} 16) \\
\mathrm{TY}(0)\end{array}$ & $\begin{array}{l}\text { SY } 4270 \\
\text { TE } 2331 \\
\text { SE } 1897 \\
\text { TY }(0)\end{array}$ & $\begin{array}{c}\mathrm{SY}=8, \mathrm{TE}=13 \\
\mathrm{SE}=9, \mathrm{TY}=0\end{array}$ \\
\hline $\begin{array}{l}\text { Age and gender } \\
\text { controlled by } \\
\text { constitution group }\end{array}$ & Age & $\begin{array}{c}\text { Age, gender } \\
\text { (female }<60 \text { yrs) }\end{array}$ & na & Gender & Age, gender \\
\hline Type of study & $\begin{array}{l}\text { Cross-sectional } \\
\text { study (2012) }\end{array}$ & $\begin{array}{l}\text { Cross-sectional study } \\
\text { (Genomic cohort 2006) }\end{array}$ & $\begin{array}{l}\text { Medical record } \\
\text { retrospective review } \\
(2008-2009)\end{array}$ & $\begin{array}{c}\text { Health } \\
\text { examination } \\
2005\end{array}$ & $\begin{array}{c}\text { Acupuncture at } \\
\text { LI4 }\end{array}$ \\
\hline MHR & na & No difference & No difference & No difference & No difference \\
\hline mRR (ms) & na & na & na & na & na \\
\hline SDNN (ms) & No difference & No difference & No difference & $\mathrm{SE}>\mathrm{TE}$ & No difference \\
\hline $\operatorname{rMSSD}(\mathrm{ms})$ & No difference & na & na & na & na \\
\hline $\mathrm{LF}\left(\mathrm{ms}^{2}\right)$ & No difference & na & No difference & na & No difference \\
\hline $\mathrm{HF}\left(\mathrm{ms}^{2}\right)$ & No difference & na & No difference & na & No difference \\
\hline LFnu & No difference & $\begin{array}{l}\text { SY }>\text { TE (all) SY > TE, SE } \\
\text { (female, below } 60 \text { years) }\end{array}$ & na & na & na \\
\hline HFnu & No difference & $\begin{array}{l}\mathrm{TE}>\mathrm{SY} \text { (all) TE, SE }>\text { SY } \\
\text { (female, below } 60 \text { years) }\end{array}$ & na & na & na \\
\hline $\operatorname{Ln}(\mathrm{LF})$ & na & No & na & na & na \\
\hline Ln (HF) & na & No & na & na & na \\
\hline $\mathrm{LF} / \mathrm{HF}$ & No difference & $\begin{array}{c}\text { SY }>\text { TESY }>\text { TE, SE } \\
(\text { female, below } 60 \text { years })\end{array}$ & $\mathrm{SY}, \mathrm{TE}>\mathrm{SE}$ & No difference & Low in TE \\
\hline
\end{tabular}

SY, Soyangin constitution; TE, Taeumin constitution; SE, Soeumin constitution; M, male; F, female; na, not available. ${ }^{1}$ Multivariated adjusted odds ratio HRV analysis. The odds ratio adjusted for age, gender, education period, marital status, drinking status, smoking status, past history (hypertension, diabetes mellitus, and hyperlipidemia), BMI, and metabolic syndrome. ${ }^{2} \mathrm{HRV}$ reporting generated indices (stress index, fatigue index) and TP showed a significant difference between constitution groups.

3.3.4. SCM and HRV after Acupuncture Stimulation $(p<0.05)$ (Table 2). 5 out of 7 SCM acupuncture studies reported some HRV differences between constitutions. Two within-subject studies $[38,41]$ reported that Taegeuk acupuncture stimulation (i.e., a predefined acupuncture formula for a specific constitution) resulted in a significant increase in HFnu in both the Tae-Eum and So-Yang type compared to a resting or stress condition, indicating a relative increase in cardiac vagal modulation. Three between-subject studies based on different acupuncture stimulation methods reported different HRV measures or conflicting data: (1) So-Yang type showed higher SDNN than So-Eum type and Tae-Eum type during passive coping conditions (i.e., enduring pain passively) and the opposite during active coping condition (i.e., pain stimulation will stop when signaling) when pain is induced by electroacupuncture [51]; (2) So-Eum type showed higher rMSSD compared to Tae-Eum type and Tae-Eum type showed higher LFnu and LF/HF compared to So-Eum type based on changes between right after needle insertion at LR3 and LI4 and 1 hour after needle removal [52]; (3) LFnu and LF/HF were increased in So-Yang type and LF/HF was significantly higher in So-Eum type compared to So-Yang type, while LF/ HF of Tae-Eum type was in between, after acupuncture stimulation at LI4 only [47].

3.3.5. SCM and HRV after Other Interventions $(p<0.05)$ (Table 5). So-Eum type had significantly enhanced HRV (i.e., SDNN) after either a meditation program [37] or an autogenic training program [48]. SDNN (time domain variable) results recorded on short-term HRV, however, may need further validation of reproducibility.

\subsection{HRV Reporting and Interpretation}

3.4.1. Reporting of HRV Measures (Table 6). The number of reported HRV variables varied from more than five $(n=9)$ to only one (e.g., SDNN or LF/HF) $(n=2)$. The most frequently reported variable was SDNN $(n=16)$, and the least was $\mathrm{mRR}$ $(n=4)$. Frequency domain variables were used to describe sympathovagal modulation: LF/HF $(n=14), \mathrm{LF}$ and HF 
TABLE 4: HRV difference by constitution $(p<0.05)$, controlled breathing.

\begin{tabular}{|c|c|c|c|}
\hline Reference & $4[36]$ & $2[34]$ & $3[35]$ \\
\hline Constitution & SCM & SCM & SCM \\
\hline Population (age) & 78 healthy students $(20-30)$ & $\begin{array}{l}32 \text { healthy students } \\
(20-30)\end{array}$ & 60 healthy students $(20-30)$ \\
\hline Subjects by constitution group & $\mathrm{SY}=13, \mathrm{TE}=30, \mathrm{SE}=35, \mathrm{TY}=0$ & $\begin{array}{c}\mathrm{SY}=10, \mathrm{TE}=11, \mathrm{SE}=11 \\
\mathrm{TY}=0\end{array}$ & $\begin{array}{c}\mathrm{SY}=18, \mathrm{TE}=18, \mathrm{SE}=24 \\
\mathrm{TY}=0\end{array}$ \\
\hline $\begin{array}{l}\text { Age and gender controlled by } \\
\text { constitution group }\end{array}$ & Age & Age & Age \\
\hline $\begin{array}{l}\text { HRV baseline difference by } \\
\text { constitution }\end{array}$ & MHR: SY, SE > TE & No difference & No difference \\
\hline $\begin{array}{l}\text { HRV by constitution compared to } \\
\text { baseline, with paced breathing }\end{array}$ & $\begin{array}{l}\text { Breath-counting on inspiration and } \\
\text { expiration (not controlling) }\end{array}$ & $\begin{array}{c}\text { Paced breathing: } 12,6 \text {, or } 3 \\
\text { times per min }\end{array}$ & $\begin{array}{l}\text { Ratio of inhalation and } \\
\text { exhalation }(4: 6,6: 4)\end{array}$ \\
\hline MHR & CIB $:$ SE $>$ TECEB $:$ SE, SY > TE & No difference & No difference \\
\hline $\mathrm{mRR}(\mathrm{ms})$ & na & No difference & No difference \\
\hline SDNN (ms) & No difference & No difference & No difference \\
\hline $\mathrm{rMSSD}(\mathrm{ms})$ & na & No difference & No difference \\
\hline $\mathrm{LF}\left(\mathrm{ms}^{2}\right)$ & No difference & na & No difference \\
\hline $\mathrm{HF}\left(\mathrm{ms}^{2}\right)$ & No difference & na & No difference \\
\hline LFnu & na & na & No difference \\
\hline HFnu & na & na & No difference \\
\hline $\operatorname{Ln}(\mathrm{LF})$ & na & No difference & na \\
\hline $\operatorname{Ln}(\mathrm{HF})$ & na & No difference & na \\
\hline $\mathrm{LF} / \mathrm{HF}$ & No difference & na & na \\
\hline
\end{tabular}

na, not available; CIB, Counting on Inspiration; CEB, Counting on Expiration.

TABLE 5: HRV difference by constitution $(p<0.05)$, associated with other interventions.

\begin{tabular}{|c|c|c|c|c|c|}
\hline Reference & 5 [37] & $10[42]$ & $14[46]$ & $16[48]$ & $18[50]$ \\
\hline Constitution & SCM & SCM & SCM & SCM & SCM \\
\hline Population & 78 students & $\begin{array}{l}47 \text { healthy subjects } \\
(29-66)\end{array}$ & $10 \mathrm{TE}$ patients & $\begin{array}{l}39 \text { patients } \\
(20-59)\end{array}$ & $\begin{array}{c}44 \text { healthy } \\
\text { subjects }(20-30)\end{array}$ \\
\hline $\begin{array}{l}\text { Subjects by constitution } \\
\text { group }\end{array}$ & na & $\begin{array}{c}\mathrm{M}(\mathrm{SY}=10, \mathrm{TE}=17 \\
\mathrm{SE}=20, \mathrm{TY}=0) \\
\mathrm{F}(\mathrm{SY}=8, \mathrm{TE}=9 \\
\mathrm{SE}=12, \mathrm{TY}=0)\end{array}$ & $\mathrm{TE}=10$ & $\begin{array}{l}\mathrm{SY}=9, \mathrm{TE}=12 \\
\mathrm{SE}=18, \mathrm{TY}=0\end{array}$ & $\begin{array}{c}S Y=10, T E=20 \\
S E=14, T Y=0\end{array}$ \\
\hline $\begin{array}{l}\text { Age and gender } \\
\text { controlled by } \\
\text { constitution group }\end{array}$ & na & Gender, age & na & na & Age \\
\hline Intervention & $\begin{array}{l}\text { Meditation } \\
\text { program }(\alpha \\
\text { version })\end{array}$ & $\begin{array}{c}\text { Forrest healing program } \\
\text { (aroma, foods, tea, } \\
\text { trekking) }\end{array}$ & $\begin{array}{l}\text { TE herbal formula } \\
\text { (Jowisengcheong-tang) }\end{array}$ & $\begin{array}{l}\text { Autogenic } \\
\text { training }\end{array}$ & $\begin{array}{l}\text { Emotional } \\
\text { stimulus (horror } \\
\text { film) }\end{array}$ \\
\hline $\begin{array}{l}\text { HRV baseline difference } \\
\text { by constitution }\end{array}$ & No difference & No difference & na & No difference & No difference \\
\hline \multicolumn{6}{|c|}{ HRV by constitution compared to baseline, associated with other interventions } \\
\hline MHR & $\begin{array}{l}\text { Decreased in SE } \\
\text { Decreased in SY }\end{array}$ & Increased in SY & na & Decreased in $\mathrm{TE}$ & na \\
\hline $\mathrm{mRR}(\mathrm{ms})$ & na & na & No difference & na & No difference \\
\hline SDNN (ms) & Increased in SE & Decreased in SY & No difference & $\begin{array}{l}\text { Increased in SE } \\
\text { Increased in } \mathrm{TE}\end{array}$ & No difference \\
\hline rMSSD (ms) & Increased in SE & No difference & No difference & na & na \\
\hline $\mathrm{LF}\left(\mathrm{ms}^{2}\right)$ & No difference & No difference & No difference & $\mathrm{Na}$ & TE $>$ SY, SE \\
\hline $\mathrm{HF}\left(\mathrm{ms}^{2}\right)$ & No difference & No difference & No difference & na & No difference \\
\hline LFnu & No difference & No difference & No difference & No difference & No difference \\
\hline HFnu & No difference & No difference & No difference & No difference & No difference \\
\hline Ln (LF) & na & na & na & na & na \\
\hline Ln (HF) & na & na & na & na & na \\
\hline $\mathrm{LF} / \mathrm{HF}$ & No difference & No difference & na & No difference & No difference \\
\hline
\end{tabular}

na, not available; M, male; F, female; SE, Soeumin constitution; SY, Soyangin constitution; TE, Taeumin constitution. 
TABLE 6: Measures of short-term HRV reporting (20 studies).

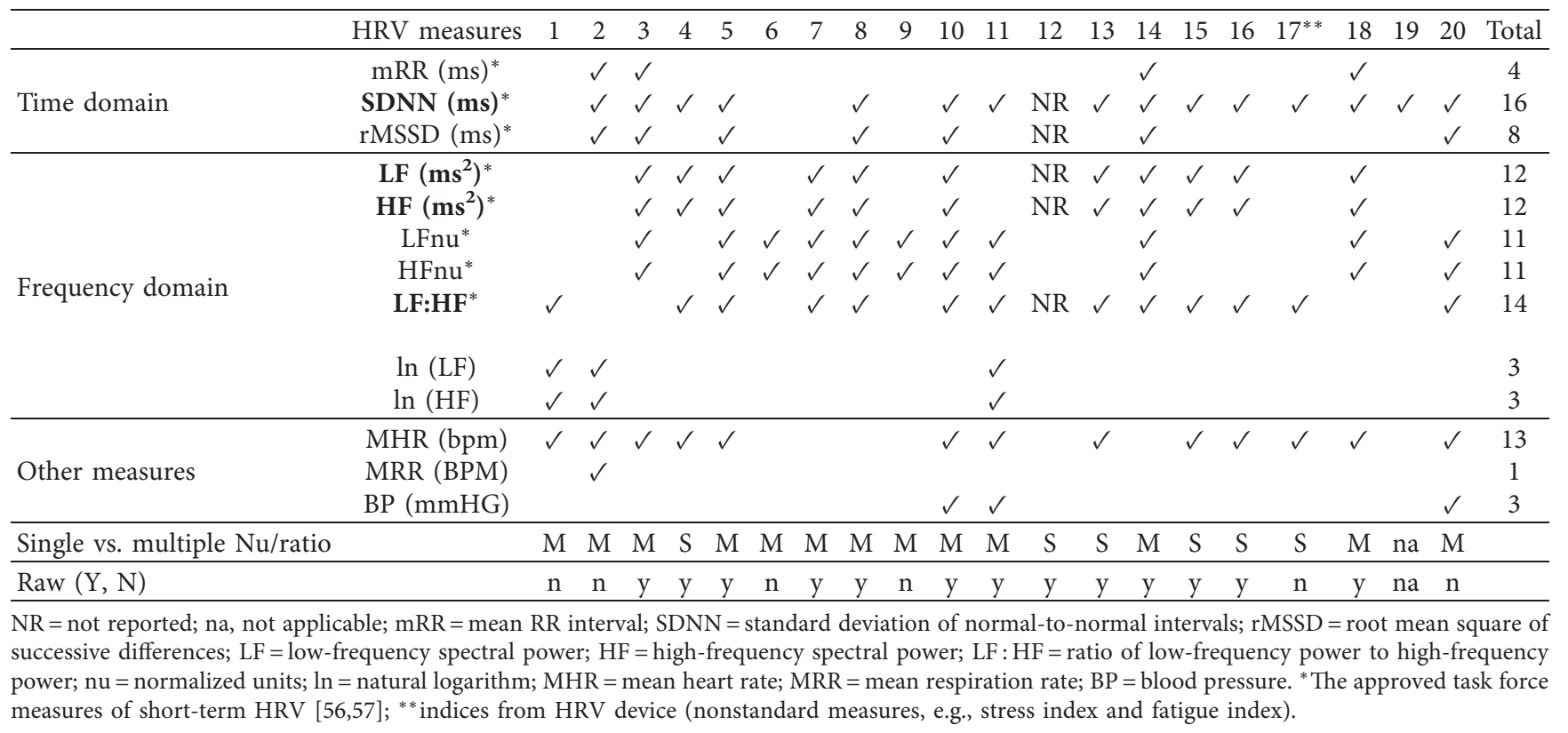

power $(n=12)$, LFnu and HFnu $(n=11)$, and natural logarithm $(n=3)$. Other HRV influencing parameters reported include mean heart rate $(n=13)$, respiration rate $(n=1)$, and blood pressure $(n=3)$.

3.4.2. Normalized Units and Raw Values (Table 7). 13 of the 20 studies reported multiple nu/ratios (i.e., HFnu, LFnu, and $\mathrm{LF} / \mathrm{HF}$ ratio), and this could present potential problems of redundancy and interpretation, especially when the HRV reporting measures provide inconsistent outcomes, as noted in Heathers' HRV methodology study [32]: for example, if LFnu was significant and LF/HF not, this might be interpreted as a change in sympathetic activity but there is no sympathovagal balance. Some SCM and HRV studies reported redundant [43] or inconsistent results: for example, LFnu increased in So-Yang type, but there is no change in HFnu [47], or HFnu was higher in So-Yang type than TaeEum type but there is no difference in LHnu [52]. While the task force recommended that research should always report both raw values and normalized units [56] because the changes in the individual frequency bands may be inconsistent with the reporting of lone normalized HRV values [32], 6 of 20 studies reported normalized units without raw values.

3.4.3. Interpretation of HF, LF, and LF: HF Ratio (Table 8). ECM and SCM studies $(n=14)$ interpreted HF as reflecting parasympathetic nervous system (PNS) mediated by RSA (Respiratory Sinus Arrhythmia) $(n=7) ; n=6$ as PNS, and $n=1$ as RSA. This mirrors the debate on LF interpretation as a mix of sympathetic and vagal, and baroreceptor activities [58], and the ECM and SCM studies $(n=14)$ showed a mixed interpretation: baroreceptor activity $(n=1)$, more SNS than PNS $(n=5)$, baroreceptor + PNS $\quad(n=1), \quad$ baroreceptor + SNS + PNS
TABLE 7: Reporting raw vs. adjusted values and single vs. multiple normalized ratio units*.

\begin{tabular}{lccc}
\hline & Raw values & No raw values & \\
\hline Single nu/ratio unit & 5 & 1 & 6 \\
Multiple nu/ratio unit & 8 & 5 & 13 \\
& 13 & 6 & $n=19$ \\
\hline
\end{tabular}

*Table format [32].

$(n=3)$, SNS + balance of PNS and SNS $(n=1)$, and index of SNS $(n=3)$. Although all the ECM and SCM studies reported LF : HF as an index of sympathovagal balance, a recent consensus suggested lowering its predictive value [58], due to the loose relationship of LF power with sympathetic outflow [32], and the nonlinear and nonreciprocal relationship between SNS and PNS activity [59]. The discrepancy in HRV interpretation is problematic in deriving a conclusive insight on the correlation between constitutions and HRV.

3.4.4. Extraneous Factors Controlled for HRV (Table 9). In general, some population variables (i.e., age, health condition, and medication) of ECM and SCM studies $(n=20)$ were well controlled $(n=14)$, but gender $(n=9)$ was relatively less controlled. Several procedure- and environment-related variables were frequently controlled (i.e., posture, resting, circadian rhythm, caffeinated drinks, alcohol, room lighting, or noise), with others less frequently controlled (i.e., smoking, wakefulness or talk, food, physical exercise, and temperature), and some not at all (i.e., bladder filling and stress level).

3.5. Classification of Constitutions. An ECM study [33] used pulse diagnosis with an intrarater reliability test (Kappa index 0.83\%). SCM studies used QSCCII (Questionnaire for 


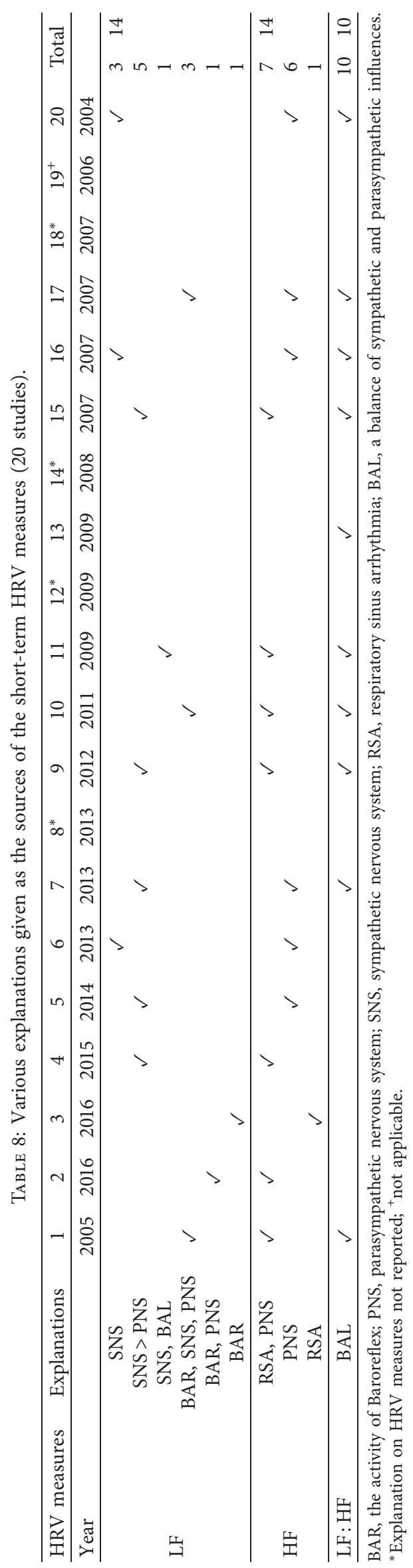


TABLE 9: Extraneous factors controlled for HRV (20 studies).

\begin{tabular}{|c|c|c|c|c|c|c|c|c|c|c|c|c|c|c|c|c|c|c|c|c|c|c|}
\hline & $\begin{array}{l}\text { Extraneous } \\
\text { factors }\end{array}$ & 1 & 2 & 3 & 4 & $5^{*}$ & 6 & 7 & 8 & 9 & $10^{*}$ & $11^{*}$ & 12 & 13 & 14 & 15 & 16 & 17 & 18 & 19 & 20 & Total \\
\hline \multirow{5}{*}{$\begin{array}{l}\text { Study } \\
\text { population }\end{array}$} & Age & & $\checkmark$ & $\checkmark$ & $\checkmark$ & & & $\checkmark$ & $\checkmark$ & $\checkmark$ & $\checkmark$ & $\checkmark$ & $\checkmark$ & & & $\checkmark$ & & & $\checkmark$ & $\checkmark$ & $\checkmark$ & 13 \\
\hline & Gender & & & & & & & $\checkmark$ & & $\checkmark$ & $\checkmark$ & $\checkmark$ & & & & $\checkmark$ & & $\checkmark$ & & $\checkmark$ & & 7 \\
\hline & $\begin{array}{l}\text { Health } \\
\text { condition }\end{array}$ & $\checkmark$ & $\checkmark$ & $\checkmark$ & $\checkmark$ & & $\checkmark$ & $\checkmark$ & $\checkmark$ & $\checkmark^{* *}$ & & $\checkmark$ & $\checkmark$ & & $\checkmark$ & $\checkmark$ & $\checkmark$ & & $\checkmark$ & $\checkmark$ & $\checkmark$ & 16 \\
\hline & Medication & $\checkmark$ & $\checkmark$ & $\checkmark$ & $\checkmark$ & & $\checkmark$ & $\checkmark$ & $\checkmark$ & $\checkmark$ & & $\checkmark$ & $\checkmark$ & & $\checkmark$ & $\checkmark$ & $\checkmark$ & & $\checkmark$ & & $\checkmark$ & 15 \\
\hline & BMI & $\checkmark$ & & & & & & & & & $\checkmark$ & $\checkmark$ & & & & & & & & & $\checkmark$ & 4 \\
\hline \multirow[t]{5}{*}{ Sample size } & & 42 & 32 & 60 & 78 & 78 & 16 & 6 & 63 & 8 & 47 & 665 & 20 & 103 & 10 & 30 & 39 & 8498 & 44 & 86 & 19 & \\
\hline & Posture & $\sqrt{ }^{1}$ & $\checkmark^{1}$ & $\checkmark^{2}$ & $\checkmark^{3}$ & & $\checkmark^{1}$ & $\checkmark^{1}$ & $\sqrt{ }^{1}$ & $\checkmark^{1}$ & & $\sqrt{ }^{3}$ & $\checkmark^{1}$ & $\checkmark^{1}$ & $\checkmark^{1}$ & $\checkmark^{1}$ & $\sqrt{ }^{3}$ & & & $\checkmark^{1}$ & $\checkmark$ & 16 \\
\hline & Resting & $\mathfrak{V}^{\mathrm{a}}$ & $\checkmark^{\mathrm{b}}$ & NA & NA & & $\checkmark^{\mathrm{b}}$ & $\begin{array}{l}\checkmark \\
b\end{array}$ & $\begin{array}{l}\checkmark \\
b\end{array}$ & $\checkmark^{b}$ & & & $\begin{array}{l}\checkmark \\
\mathrm{b}\end{array}$ & $\checkmark^{b}$ & $\checkmark^{c}$ & $\checkmark^{\mathrm{a}}$ & $\checkmark^{\mathrm{d}}$ & & $\begin{array}{l}\checkmark \\
\mathrm{a}\end{array}$ & $\checkmark^{\mathrm{b}}$ & & 13 \\
\hline & $\begin{array}{l}\text { Circadian } \\
\text { rhythm }\end{array}$ & & $\checkmark$ & $\checkmark$ & $\checkmark$ & & $\checkmark$ & $\checkmark$ & & $\checkmark$ & & & $\checkmark$ & & $\checkmark$ & $\checkmark$ & & & & & $\checkmark$ & 10 \\
\hline & $\begin{array}{l}\text { Wakefulness, } \\
\text { talk }\end{array}$ & & & $\checkmark$ & $\sqrt{ }$ & & & & & & & & & & & $\checkmark$ & $\checkmark$ & & $\checkmark$ & $\checkmark$ & $\checkmark$ & 7 \\
\hline \multirow[t]{5}{*}{ Study procedure } & $\begin{array}{l}\text { Caffeinated } \\
\text { drinks }\end{array}$ & $\checkmark$ & $\checkmark$ & $\checkmark$ & $\sqrt{ }$ & & $\checkmark$ & $\checkmark$ & & $\checkmark$ & & & & $\checkmark$ & & $\checkmark$ & $\checkmark$ & & & & & 10 \\
\hline & Alcohol & $\checkmark$ & $\checkmark$ & $\checkmark$ & $\checkmark$ & & $\checkmark$ & $\checkmark$ & & $\checkmark$ & & & & $\checkmark$ & & $\checkmark$ & $\checkmark$ & & & & & 10 \\
\hline & Smoking & $\checkmark$ & & & & & $\checkmark$ & $\checkmark$ & & $\checkmark$ & & & & $\checkmark$ & & $\checkmark$ & $\checkmark$ & & & & & 7 \\
\hline & $\begin{array}{c}\text { Food } \\
\text { Bladder filling }\end{array}$ & $\checkmark$ & & $\checkmark$ & & & $\checkmark$ & $\checkmark$ & & $\checkmark$ & & & & & & $\checkmark$ & & & & & & $\begin{array}{l}6 \\
0\end{array}$ \\
\hline & $\begin{array}{c}\text { Physical exercise } \\
\text { Stress level }\end{array}$ & & & & & & $\checkmark$ & $\checkmark$ & & $\checkmark$ & & & & & & & & & & & & $\begin{array}{l}3 \\
0\end{array}$ \\
\hline \multirow{3}{*}{$\begin{array}{l}\text { Study } \\
\text { environment }\end{array}$} & Light & $\checkmark$ & & & & & $\checkmark$ & $\checkmark$ & $\checkmark$ & $\checkmark$ & & & $\checkmark$ & & $\checkmark$ & & & & $\checkmark$ & $\checkmark$ & & 9 \\
\hline & Noise & $\checkmark$ & $\checkmark$ & $\checkmark$ & $\checkmark$ & & $\checkmark$ & $\checkmark$ & $\checkmark$ & $\checkmark$ & & & $\checkmark$ & & $\checkmark$ & $\checkmark$ & & & $\checkmark$ & $\checkmark$ & & 13 \\
\hline & Temperature & $\checkmark$ & & & & & & & & & & & & & & & & & $\checkmark$ & & & 2 \\
\hline
\end{tabular}

$\begin{array}{llllllllllllllllllllllll}\text { Factors } & 12 & 9 & 10 & 10 & 0 & 12 & 14 & 7 & 14 & 1 & 6 & 8 & 5 & 7 & 13 & 8 & 1 & 8 & 8 & 7\end{array}$

total $=19$

NA, not applicable; ${ }^{\mathrm{a}} 10 \mathrm{~min}$ resting before HRV; ${ }^{\mathrm{b}} 5 \mathrm{~min}$ resting before HRV; ${ }^{\mathrm{c}}$ resting time not available; ${ }^{\mathrm{d}} 1 \mathrm{~min}$ resting before HRV; ${ }^{1}$ supine; ${ }^{2}$ sitting and standing; ${ }^{3}$ sitting; ${ }^{*}$ information on HRV control factors not available; ${ }^{* *}$ menstruation factor controlled; ${ }^{+}$multivariated adjusted odds ratio analysis (age, gender, BMI, alcohol drinking status, smoking status, health condition, metabolic syndrome, marital status, and education level); 9 am to 4 pm (wide range).

Sasang Constitution Classification) ( $n=9)$, practitioner diagnosis based on SCAT (i.e., Sasang Constitution Analysis Tool including facial, voice, body, and QSCCII) $(n=6)$, practitioner diagnosis based on QSCCII $(n=4)$, and practitioner only diagnosis $(n=2)$.

\section{Discussion}

This systematic review explored HRV as a biomedical diagnostic for the Disympathetic Dimension of ECM.

4.1. Limitations of the Study. There are limitations to this review. The focus was on a qualitative and descriptive analysis of ECM and SCM studies on HRV reporting, interpretation, and control of extraneous factors. A review of statistical analysis including study population and effect size calculation was not within the study scope. Most articles were derived from the Korean research literature; despite the care with translation, misinterpretation or misunderstanding of the study contents is possible.

4.2. Correlation between HRV and Constitution. The results of the systematic review showed little consistency in the data to support the use of HRV as an objective determinant of ECM constitutions.

(1) A single ECM study of HRV differences after eightconstitution acupuncture had several limitations: sample size, control of age, and gender factors, and the data was not sufficient to draw meaningful conclusions on the use of HRV for constitutional differentiation along the Disympathetic Dimension

(2) While consensus exists for HF as a proxy to evaluate cardiac vagal modulation when the respiratory frequency is mediated, LF and the LF/HF ratio lack a clear relationship to cardiac sympathetic modulation. None of the ECM and SCM studies reported significant differences between constitutions when measuring HF at resting. Two SCM studies showed some constitutional differences in the $\mathrm{LF} / \mathrm{HF}$ ratio; however, the ratio lacks consensus as a reliable measure for sympathovagal balance $[29,30,56]$. The results alone, therefore, are not enough to explain the constitutional differences in terms of cardiac autonomic modulation.

(3) While constitutional differences in HRV measures (i.e., SDNN, HFnu, LFnu, and LF/HF) in the SCM acupuncture stimulation studies are notable, there 
were limitations: HRV time domain values such as SDNN [21] are preferably computed through longterm recording ( 24 hours); therefore, the study result based on 30 seconds of SDNN requires further validation of reproducibility; LF/HF and LFnu are not sufficient to reflect cardiac autonomic modulation and changes in those measures alone have limited predictive value of constitutional differences.

(4) While 5 out of 7 SCM acupuncture studies reported some HRV differences (HFnu, LFnu, LF/HF, and SDNN), the variety of study methods and procedure design made it difficult to compare, consolidate, and draw a robust conclusion. This variety includes: reporting of HRV measures (e.g., HFnu, LFnu, LF/HF, SDNN, and rMSSD), acupuncture methods and points (e.g., Taegeuk acupuncture, bee venom acupuncture, electroacupuncture at ST36 and ST38, acupuncture at LI4 or LI4 and LR3), frequency and duration (e.g., one session vs. three sessions over two weeks, $5 \mathrm{~min}$ vs. 15 min acupuncture), stimulation methods (e.g., only acupuncture vs. mental stress and acupuncture), study population (e.g., age, gender), HRV measurement timing (e.g., right after needle removal, 1 hour after needle removal), and control of extraneous factors (e.g., wakefulness or talk, food).

4.3. HRV Reporting and Interpretation. HRV reporting in the studies showed some opportunities to improve: inconsistency in the selection of HRV reporting measures, redundancy or inconsistent outcomes of normalized unit reporting (i.e., HFnu, LFnu, and LF/HF ratio) without raw values, and discrepancy in HRV interpretation (HF, LF, and LF/HF ratio). ECM and SCM studies reported only some of the HRV measures (i.e., $\mathrm{mRR}, \mathrm{SDNN}$, rMSSD, LF power, HF power, LFnu, HFnu, and LF:HF) that were recommended by a task force $[58,59]$ and the selection of measures were also inconsistent among the studies.

4.4. Extraneous Factors. Among the HRV extraneous factors, some of the population variables (i.e., age, health condition, and medication) were well controlled, but gender and other procedural variables (e.g., wakefulness or talk, food) were less controlled in the studies.

In the studies examined, there was no clear relationship between HRV and Korean Constitutional Medicine, including the Disympathetic Dimension of ECM. Reasons included demographic discrepancies (i.e., age, gender, and health conditions), HRV reporting, methodological inconsistencies between the SCM studies, and insufficient ECM research. The continuing debates on whether HRV measures reflect autonomic function accurately add further complications on top of HRV's sensitivity to various extraneous factors.

\section{Conclusions}

This review examined HRV in the hope that it would be a useful objective diagnostic tool to bridge the information gap for acupuncture and traditional medicine researchers and, specifically, for determining a patient's position on the Disympathetic Dimension of Eight-Constitution Medicine. HRV does not seem to be suitable for this purpose alone.

\author{
Abbreviations \\ ECG: Electrocardiogram \\ ECM: Eight-constitution medicine \\ HF: High frequency \\ HFnu: Normalized high frequency \\ HRV: Heart rate variability \\ IBI: Interbeat intervals \\ KCM: Korean constitution medicine \\ LF: $\quad$ Low frequency \\ LFnu: Normalized low frequency \\ LI4: $\quad$ Large intestine 4 \\ LR3: $\quad$ Liver 3 \\ mRR: $\quad$ Mean of R-R intervals \\ SCM: Sasang constitution medicine \\ SDNN: Standard deviation of NN intervals \\ PPG: Photoplethysmogram \\ rMSSD: Root mean square of the successive differences.
}

\section{Data Availability}

The datasets used and analyzed during the current study are available from the corresponding author on reasonable request.

\section{Disclosure}

The role of the funding body in the design of the study includes collection, analysis, and interpretation of data and in writing the manuscript.

\section{Conflicts of Interest}

The authors declare that they have no conflicts of interest.

\section{Authors' Contributions}

HK and SW designed the study. HK conducted the systematic review and drafted the manuscript. SW, BO, and TR reviewed and edited the manuscript. BJC provided advice on ECM. All authors approved the final manuscript.

\section{Acknowledgments}

The lead author and this work were supported by an internal Higher Degree Research (HDR) grant (University of Technology Sydney).

\section{References}

[1] T. Kim, "Tradition on the move," Asian Medical, vol. 11, no. 1-2, pp. 133-159, 2016.

[2] D. Kuon, "Constitution-acupuncture," Journal of Acupuncture - Moxibustion, vol. 10, pp. 149-167, 1965. 
[3] H. J. Kim, B. H. Jang, M. J. Kim, K. C. Kim, W. J. Kuon, and C. K. Kim, "Prevalence of and associations between metabolic syndrome and the constitutions defined by Korean Eight Constitution Medicine," Medicine (Baltimore), vol. 99, no. 7, p. e19074, 2020.

[4] H. Chae, I. K. Lyoo, S. J. Lee et al., "An alternative way to individualized medicine: psychological and physical traits of Sasang typology," The Journal of Alternative and Complementary Medicine, vol. 9, no. 4, pp. 519-528, 2003.

[5] D. Kuon, "Eight-constitution medicine: an overview," IMKS Occas Pap No2, vol. 12, pp. 601-623, 1999.

[6] O. Golubnitschaja, J. Kinkorova, and V. Costigliola, "Predictive, Preventive and Personalised Medicine as the hardcore of "Horizon 2020": EPMA position paper," EPMA Journal, vol. 5, no. 1, p. 6, 2014.

[7] J. Bland, "Functional medicine: An operating system for integrative medicine," Journal of Integrative Medicine, vol. 14, no. 5, pp. 18-20, 2015.

[8] A. Ali, "Disease prevention and health promotion: how integrative medicine fits," American Journal of Preventive Medicine, vol. 49, no. 5, pp. S230-S240, 2015.

[9] C. Yin, H.-J. Park, Y. Chae et al., "Korean acupuncture: the individualized and practical acupuncture," Neurological Research, vol. 29, no. sup1, pp. 10-15, 2007.

[10] M. J. Paik, D. Kuon, J. Cho, and K.-R. Kim, "Altered urinary polyamine patterns of cancer patients under acupuncture therapy," Amino Acids, vol. 37, no. 2, pp. 407-413, 2009.

[11] Y.-S. Kim, H. Jun, Y. Chae et al., "The practice of Korean medicine: an overview of clinical trials in acupuncture," Evidence-Based Complementary and Alternative Medicine, vol. 2, no. 3, pp. 325-352, 2005.

[12] K. Emma and W. Sean, Pulse Diagnosis: A Clinical Guide\|, Elsevier Health Sciences, Amsterdam, Netherlands, 2008.

[13] H.-S. Lee, Y.-B. Lee, Y.-S. Shin et al., "A pilot study on reliability of pulse diagnosis in Eight-Constitution Medicine," Korean Journal Acupuncture, vol. 22, no. 4, pp. 1-8, 2005.

[14] Y.-S. Shin, Y.-J. Park, H.-S. Oh, and Y.-B. Park, "A study on method that estimate expertness of pulse diagnosis in 8 Constitution Medicine," JournalKorea Institute Orientied Medical Diagnostics, vol. 10, 2006.

[15] Y.-S. Shin, S.-S. Nah, H.-S. Oh, Y.-J. Park, and Y.-B. Park, “A study on consistency and accuracy of pulse diagnosis in EightConstitution Medicine," Oriental Pharmacy and Experimental Medicine, vol. 9, no. 1, pp. 14-19, 2009.

[16] M. S. Lee, B.-C. Shin, S.-M. Choi, and J. Y. Kim, "Randomized clinical trials of constitutional acupuncture: a systematic review," Evidence-Based Complement Alternative Medicine, vol. 6, no. S1, pp. 59-64, 2009.

[17] P. Eckman, The Compleat Acupuncturist: A Guide to Constitutional and Conditional Pulse Diagnosis, Singing Dragon, London, UK, 2014.

[18] H. Eppinger, L. Hess, W. M. Kraus, and S. E. Jelliffe, Vagotonia: A Clinical Study in Vegetative Neurology, Nervous and Mental Disease Publishing Company, 1915.

[19] J. B. Cohen, "Tests for Disclosing Sympathicotonia and Vagotonia.*," Anesthesia \& Analgesia, vol. 5, no. 4, 1926.

[20] F. Lombardi and P. K. Stein, "Origin of heart rate variability and turbulence: an appraisal of autonomic modulation of cardiovascular function," Frontier Physiology, vol. 295 pages, 2011.

[21] R. Freeman, "Assessment of cardiovascular autonomic function," Clinical Neurophysiology, vol. 117, no. 4, pp. 716730, 2006.
[22] G. Ernst, Heart Rate Variability, Springer, Berlin, Germany, 2016.

[23] M. J. Ferreira Jr and A. Zanesco, "Heart rate variability as important approach for assessment autonomic modulation," Motriz: Revista de Educação Física, vol. 22, no. 2, pp. 3-8, 2016.

[24] D. S. Quintana, G. A. Alvares, and J. A. J. Heathers, "Guidelines for reporting articles on psychiatry and heart rate variability (GRAPH): recommendations to advance research communication,” Translational Psychiatry, vol. 6, 2016.

[25] S. Michael, K. S. Graham, and G. M. Davis, "Cardiac autonomic responses during exercise and post-exercise recovery using Heart Rate Variability and Systolic Time Intervals-A Review," Frontier Physiology, vol. 8, no. May, pp. 1-19, 2017.

[26] G. E. Billman, "The effect of heart rate on the heart rate variability response to autonomic interventions," Frontiers in Physiology, vol. 4, no. 222, p. 222, 2013.

[27] G. E. Billman, "Heart rate variability - A historical perspective," Frontier Physiology, vol. 2, 2011.

[28] D. S. Goldstein, O. Bentho, M. Y. Park, and Y. Sharabi, "Lowfrequency power of heart rate variability is not a measure of cardiac sympathetic tone but may be a measure of modulation of cardiac autonomic outflows by baroreflexes," Experimental Physiology, vol. 96, 2011.

[29] D. S. Quintana and J. A. J. Heathers, "Considerations in the assessment of heart rate variability in biobehavioral research," Frontier Physiology, vol. 5, 2014.

[30] G. E. Billman, H. V. Huikuri, J. Sacha, and K. Trimmel, “An introduction to heart rate variability: methodological considerations and clinical applications," Frontiers in Physiology, vol. 6, p. 55, 2015.

[31] F. Shaffer and J. P. Ginsberg, "An overview of heart rate variability metrics and norms," Frontiers in Physiology, vol. 5, 2017.

[32] J. A. J. Heathers, "Everything Hertz: methodological issues in short-term frequency-domain HRV," Frontiers in Physiology, vol. 5, p. 177, 2014.

[33] Y.-S. Shin, Y.-J. Park, H.-S. Oh, S.-C. Lee, and Y.-B. Park, "Effects of 8 Constitution Acupuncture on Heart Rate Variability," Journal Korea Institute Oriential Medical Diagnostics, vol. 9, 2005.

[34] J.-H. Kim, B.-S. Kim, S.-S. Park, Y.-J. Lee, N.-R. Keum, and H.-S. Bae, "The effects of paced breathing in specific respiration rate on heart rate variability: a pilot study," Journal of Sasang Constitutional Medicine, vol. 28, no. 2, pp. 123-131, 2016.

[35] J.-H. Kim, H.-S. Bae, and S.-S. Park, "The effects of ratio of inhalation and exhalation and posture on heart rate variability according to sasang constitution," Journal of Sasang Constitutional Medicine, vol. 28, no. 1, pp. 40-50, 2016.

[36] H.-S. Bae, J.-H. Kim, Y.-J. Lee, H.-B. Son, and S.-S. Park, "The effects of breath-counting meditation on sasang constitution," Journal of Sasang Constitutional Medicine, vol. 27, no. 2, pp. 231-239, 2015.

[37] G.-W. Kim, H.-S. Bae, H.-B. Son, P.-W. Lee, B.-S. Kim, and S.-S. Park, "Research on change of heart rate variability and psychological scale by sasang constitution according to before and after of the meditation programs ( $\alpha$ version)," Journal of Oriental Neuropsychiatry, vol. 25, no. 1, pp. 1-12, 2014.

[38] M. Oyungerel, N. S. Kim, J. Y. Kim, Y. S. Kim, and S. S. Nam, "Effects of acupuncture on the autonomic nervous system by analyzing heart rate variability in taeeumin," Acupuncture, vol. 30, no. 4, pp. 87-94, 2013. 
[39] N. S. Kim, J. Y. Kim, S. G. Kwak, I. H. Shin, S. S. Nam, and Y. S. Kim, "Effects of Taegeuk acupuncture on the autonomic nervous system by analyzing heart rate variability in 20's Soeumin," Journal Korean Acupuncture Moxibustion, vol. 30, no. 3, pp. 39-49, 2013.

[40] K. Kim, Y.-J. Ha, S.-J. Park, N.-R. Choi, Y.-S. Lee, and J.-C. Joo, "Characteristics of fatigue in Sasang constitution by analyzing questionnaire and medical devices data," Journal of Sasang Constitutional Medicine, vol. 25, no. 4, pp. 306-319, 2013.

[41] N.-S. Kim, S.-J. Kim, H.-J. Ryu, S.-S. Nam, and Y.-S. Kim, "Effects of Taegeuk acupuncture on the autonomic nervous system by analyzing heart rate variability in Soyangin," Acupunct, vol. 29, 2012.

[42] S.-G. Hong, E.-J. Choi, S.-H. Sun et al., "Biological change by forest healing according to sasang Constitution: preliminary study," Journal of Sasang Constitutional Medicine, vol. 23, no. 4, pp. 487-502, 2011.

[43] S. Kim, S. Sun, J. Yoo, S. Koh, and J. Park, "Correlation between sasang constitution and heart rate variability in wonju rural population," Journal Internal Korean Medicine, vol. 30, no. 3, pp. 510-524, 2009.

[44] S.-M. Lee, K. Kim, S.-Y. Oh, Y.-M. Kwon, and J.-C. Joo, "Effects of bee venom acupuncture on heart rate variability, pulse wave, and cerebral blood flow for types of Sasang Constitution," Journal of Korean Institute of Herbal Acupuncture, vol. 12, no. 1, pp. 35-42, 2009.

[45] C. Y. Kim, N. H. Kown, Y. J. Shin et al., "Facial electromyography and heart rate variability values of idiopathic facial palsy inpatients in relationship with sasang constitutional characteristics," Journal Korean Acupuncture Moxibustion, vol. 26, no. 6, pp. 111-119, 2009.

[46] S.-Y. Oh, S.-W. Lee, E.-Y. Kil, and J.-C. Joo, "Effects of jowisengcheong-tang on heart rate variability," Journal of Sasang Constitutional Medicine, vol. 20, no. 2, pp. 53-57, 2008.

[47] I.-H. Im, Effect of Acupuncture at LI4(Hapkok) on Heart Rate Variability in Healthy Subjects According to Sasang Constitution, Graduate School Kyung Hee University, Seoul Korea, 2007.

[48] J.-H. Lee and J.-G. Lee, "The effects of autogenic training on heart rate variability," Journal Oriential Neuropsychiatry, vol. 18, no. 1, pp. 123-132, 2007.

[49] J.-H. Lee, E.-H. Seo, J.-H. Ha, A.-R. Choi, C.-H. Woo, and D.-M. Goo, "A study on the sasang constitutional differences in heart rate variability," Journal of Sasang Constitutional Medicine, vol. 19, no. 3, pp. 176-187, 2007.

[50] G.-R. Lee, D.-Y. Shin, Y.-W. Kim, J.-H. Yi, J.-M. Song, and L.-H. Kim, "Changes of HRV according to emotional stimulus in sasang constitutional groups," Journal Oriential Neuropsychiatry, vol. 18, no. 2, pp. 25-34, 2007.

[51] K.-S. Jang, J. K. Kim, and S. K. Lee, "Analysis of heart rate variability in constitution types during active and passive coping caused dy electroacupuncture," Journal of Physiology \& Pathology in Korean Medicine, vol. 20, no. 1, pp. 115-124, 2006.

[52] C. K. Kwak, E. H. Sohn, E. J. Lee, B. H. Koh, I. B. Song, and W. Hwang, "A study about Sasang constitutional difference on autonomous function after acupuncture stimulation," Journal of Sasang Constitutional Medicine, vol. 16, no. 3, pp. 76-84, 2004.

[53] J.-H. Lee, E.-H. Seo, J.-H. Ha, A.-R. Choi, C.-H. Woo, and D.-M. Goo, "A study on the Sasang constitutional differences in heart rate variability," Journal of Sasang Constitutional Medicine, vol. 19, no. 3, pp. 176-187, 2007.

[54] J.-H. Kim and S.-S. Park, "The effects of posture and the ratio of inhalation and exhalation on heart rate variability," Journal of Korean Medicine, vol. 37, no. 1, pp. 114-124, 2016.

[55] Y. R. Han, H. B. Lee, S. Y. Han, B. J. Kim, S. J. Lee, and H. Chae, "Systematic review of type-specific pathophysiological symptoms of Sasang typology," Integrative Medicine Research, vol. 5, no. 2, pp. 83-98, 2016.

[56] M. Malik, "Heart rate variability," Annals of Noninvasive Electrocardiology, vol. 1, no. 2, pp. 151-181, 1996.

[57] D. Nunan, G. R. H. Sandercock, and D. A. Brodie, "A quantitative systematic review of normal values for short-term heart rate variability in healthy adults," Pacing Clinical Electrophysiology, vol. 33, no. 11, pp. 1407-1417, 2010.

[58] S. Laborde, E. Mosley, and J. F. Thayer, "Heart rate variability and cardiac vagal tone in psychophysiological research - recommendations for experiment planning, data analysis, and data reporting," Frontiers in Physiology, vol. 8213 pages, 2017.

[59] G. Billman, "The LF/HF ratio does not accurately measure cardiac sympatho-vagal balance," Frontiers in Physiology, vol. 4 , p. $26,2013$. 